\title{
THERMODYNAMIC ANALYSIS OF AS-CAST AND HEAT-TREATED MICROSTRUCTURES OF Mg-Ce-Nd ALLOYS
}

\author{
Mark A. Easton ${ }^{1}$, Suming Zhu ${ }^{1}$, Mark A. Gibson ${ }^{2}$, Jian-Feng Nie ${ }^{1}$, Joachim Gröbner ${ }^{3}$, Artem Kozlov ${ }^{3}$, Rainer Schmid- \\ Fetzer $^{3}$ \\ ${ }^{1}$ CAST CRC, Monash University; Victoria 3800, Australia \\ ${ }^{2}$ CAST CRC, CSIRO; Clayton, Victoria 3169, Australia \\ ${ }^{3}$ Clausthal University of Technology; Robert-Koch-Straße 42, Clausthal-Zellerfeld, D-38678, Germany
}

Keywords: Rare earth, CALPHAD, Casting, Isothermal heat treatments

\begin{abstract}
The phase relationships in the Mg-rich corner of the $\mathrm{Mg}-\mathrm{Ce}-\mathrm{Nd}$ system have been investigated through the evaluation of selected compositions in the as-cast and heat-treated condition. Consistent thermodynamic CALPHAD-type assessments have also been generated for the Mg-Ce-Nd system [1].

It is shown that this system reveals a significant degree of meta-stability under technologically relevant solidification conditions (i.e., permanent-mould or high pressure die casting). This is simulated in thermodynamic calculations by suppression of the $\mathrm{RE}_{5} \mathrm{Mg}_{41}$ phase and reasonable agreement is found with the as-cast microstructures. After heat treatment these microstructures transform, depending on alloy composition, into phase assemblies consistent with the calculated stable equilibrium phase diagram. It is the elucidation of such meta-stable phase formation and the subsequent transformation from the as-cast to the heat treated state that is a particular strength of the thermodynamic approach and which makes it a powerful tool for alloy development.
\end{abstract}

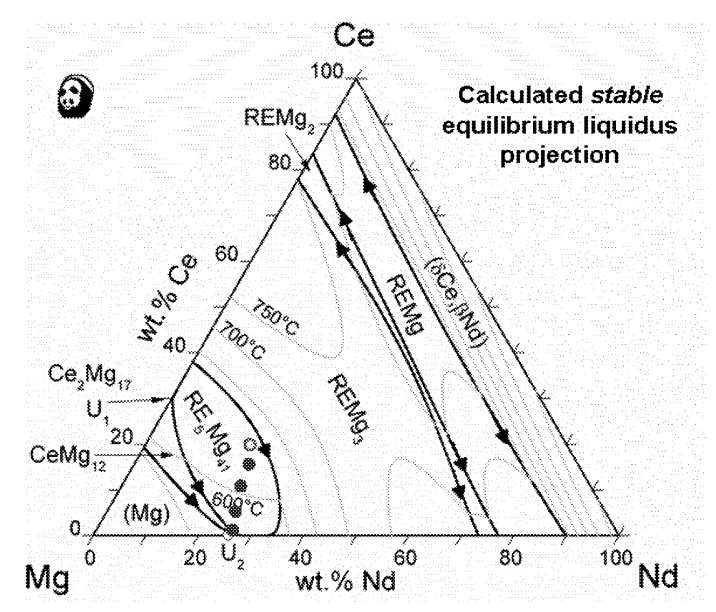

Figure 1. Mg-Ce-Nd Liquidus surface.

The solid dots in Figure 1 indicate alloys exhibiting experimentally evidenced primary precipitation of the $\mathrm{CeMg}_{12}$ phase in the as-cast condition, whereas the stable equilibrium phase diagram suggests $\mathrm{RE}_{5} \mathrm{Mg}_{41}$ to be the primary phase. The thermodynamic calculation, however, reveals that a relatively small undercooling will result in the (metastable) primary $\mathrm{CeMg}_{12}$ phase precipitation in all these alloys, as observed after casting. More results and the phase transformations towards equilibrium after heat treatment are given in detail by Gröbner et al. [1].

\section{References}

[1] J. Gröbner, A. Kozlov, R. Schmid-Fetzer, M. A. Easton, S. Zhu, M. A. Gibson, J.-F. Nie, Acta Materialia, 2010, doi:10.1016/j.actamat.2010.09.066 (in press). 